\title{
Update on female sterilisation: report from an international symposium on considerations for assessing long-term failure rates
}

\author{
Shaughn O’Brien, Janesh Gupta, Salim Najia, Mohamed Yehia
}

\section{Introduction}

The 6th International Scientific Meeting of the Royal College of Obstetricians and Gynaecologists (RCOG) took place in September 2005 in Cairo, Egypt. During the meeting, a sponsored symposium entitled 'The changing face of female sterilisation: meeting the needs of the $21 \mathrm{st}$ century woman' (sponsored by Femcare-Nikomed Ltd, manufacturer of the Filshie clip) provided an overview of the different methods of long-term contraception with a focus on female sterilisation. Here we report the main observations of the symposium, including recommendations for factors that should be considered when assessing the long-term failure rates associated with female sterilisation.

Over the past few decades, rapid advances in technology have allowed the development of a number of different contraceptive methods that are available for use today. The choice of contraception is influenced by a number of factors, including age, sexual lifestyle, relationship type, family status and medical history. In addition, as part of the necessary counselling of any family planning programme, it is essential that provision of unbiased accurate information be provided so that women can make informed decisions on long-term contraception.

Long-acting reversible contraception (LARC) methods include intrauterine devices (IUDs), the progestogen intrauterine hormone releasing system (IUS), and progestogen-only injectables and subdermal implants. In the UK, a recent National Institute for Health and Clinical Excellence (NICE) ${ }^{1}$ analysis compared the efficacy of these contraceptive methods, the findings of which showed high efficacy across all these procedures (IUD failure rate $<2 \%$ at 5 years; IUS failure rate $<1 \%$ at 5 years; injectables $<4 / 1000$ over 2 years; subdermal implants $1 / 1000$ at 3 years). These efficacies are superior to those of the two most common contraceptives used in the UK, namely hormonal oral contraception (combined and progestogen only, 50/1000 in the first year with typical use) and the

J Fam Plann Reprod Health Care 2008; 34(1): 13-18

(Accepted 15 May 2007)

Department of Obstetrics and Gynaecology, Keele University Medical School, Keele, UK

Shaughn O'Brien, MD, FRCOG, Professor of Obstetrics and

Gynaecology

Department of Obstetrics and Gynaecology, University of Birmingham, Birmingham Women's Hospital, Birmingham, UK

Janesh Gupta, MD, FRCOG, Professor of Obstetrics and Gynaecology

Department of Obstetrics and Gynaecology, Queen's Park Hospital, Blackburn, UK

K Salim Najia, MD, Consultant in Obstetrics and Gynaecology

Department of Obstetrics and Gynaecology, Ain Shams

University, Cairo, Egypt

Mohamed Yehia, MD, FRCOG, Professor of Obstetrics and

Gynaecology

Correspondence to: Professor Shaughn O'Brien, Department of Obstetrics and Gynaecology, Keele University Medical School, Keele ST5 5BG, UK. E-mail: Shaughn.obrien@uhns.nhs.uk condom (150/1000 in the first year with typical use) where effectiveness depends on their correct and consistent use. ${ }^{1}$ In terms of long-term contraception, female sterilisation represents one of the most popular long-term contraceptive methods in the world. ${ }^{2,3}$ In the UK, an estimated 50000 women undergo this procedure every year. ${ }^{4}$ Furthermore, information collected through the General Household Survey in the UK for the period 1986-1993 shows that around one in four women or their partners rely on sterilisation for family planning and, by the age of 40 years, this figure is nearly one in two. ${ }^{5}$ Despite its use as the most popular long-term contraceptive method in the world, female sterilisation attracts little publicity. Moreover, interpretations of long-term comparative data have meant that the overall efficacy of female sterilisation is often inaccurately represented.

\section{History of female sterilisation}

Tubal ligation was first proposed by James Blundell in the early 19th century. However, it was not until 1930 that the Pomeroy technique was published posthumously in the New York State Journal of Medicine. ${ }^{6}$ The Pomeroy technique, still widely used today, is a version of partial salpingectomy, which involves ligating a small loop of the Fallopian tube and cutting off the top segment of the loop. A few years later, in 1936, the first laparoscopic tubal occlusion as a method of sterilisation was performed. By the mid-20th century, laparoscopic female sterilisation began to gain in popularity. In particular, because it could be performed on a day case basis, it became popular for medical and socioeconomic reasons.

The high incidence of thermal and electrical injuries with unipolar and bipolar cautery prompted the introduction of a number of mechanical devices during the 1970s, including the Falope ring, Hulka clip, Bleier clip, Tupla clip and Filshie clip. These simplified procedures, combined with their ability to be performed in ambulatory settings, have helped minimise complications, with the result that serious complications are rare.7,8 Indeed, recent studies have reported an overall complication rate of between 4.6 to 5.5 per 1000 laparoscopic sterilisations. ${ }^{8,9}$ Advances in hysteroscopic approaches have also been made. A range of hysteroscopic methods of sterilisation has been used for a number of years, including silver nitrate thermal cautery, cryocautery and cornual plugs. ${ }^{10}$ Two recently introduced techniques, the Essure ${ }^{\circledR}$ and the Adiana methods, ${ }^{11,12}$ have also shown promising results. The surgical techniques associated with these devices are reported below.

\section{Female sterilisation techniques}

Female sterilisation techniques involve procedures for gaining access to and occluding the Fallopian tubes (Table 1). Procedures for gaining access to the Fallopian tubes are primarily abdominal, and include minilaparotomy, laparoscopy and laparotomy, which are performed under local or general anaesthesia. Laparoscopy is the preferred route for interval procedures, whereas minilaparotomy is used for postpartum patients. While laparoscopy requires more sophisticated training and equipment, minilaparotomy requires only basic surgical skills and equipment. Despite postpartum sterilisation 
Table 1 Methods of female sterilisation

\begin{tabular}{|c|c|c|c|}
\hline $\begin{array}{l}\text { Methods for } \\
\text { gaining access to } \\
\text { Fallopian tube }\end{array}$ & $\begin{array}{l}\text { Occlusion } \\
\text { procedures }\end{array}$ & Devices & Technique \\
\hline \multirow{4}{*}{$\begin{array}{l}\text { Minilaparotomy } \\
\text { Laparoscopy } \\
\text { Laparotomy }\end{array}$} & $\begin{array}{l}\text { Partial } \\
\text { salpingectomy }\end{array}$ & - & $\begin{array}{l}\text { Tying a small loop in the Fallopian tube and cutting off top } \\
\text { segment of tube }\end{array}$ \\
\hline & $\begin{array}{l}\text { Electrocoagulation } \\
\text { (unipolar, bipolar) }\end{array}$ & - & Electrical current applied to Fallopian tubes \\
\hline & Silicone rings & Falope & Silicone rubber band fitted around the Fallopian tube \\
\hline & Clips & Hulka, Bleier, Tupla, Filshie & Placement of clip on mid-isthmic portion of the Fallopian tube \\
\hline Hysteroscopic & $\begin{array}{l}\text { Silver nitrate thermal } \\
\text { cautery, cryocautery, } \\
\text { corneal plugs }\end{array}$ & Adiana, Essure ${ }^{\circledR}$ & $\begin{array}{l}\text { Rod or plug placed in Fallopian tube. Requires a } \\
\text { hysterosalpingogram to confirm device placement }\end{array}$ \\
\hline
\end{tabular}

being popular in the developing world and in the USA, it has been largely discouraged in the UK. This is thought to be the result of the perception that sterilisation conducted during this time period will have a higher failure rate over interval procedures. ${ }^{13}$ In addition, there appears to be an increased regret rate with postpartum sterilisation. ${ }^{14}$ In contrast, hysteroscopic methods offer the advantage of being performed on a day care basis and since there is no abdominal incision, little or no anaesthetic is required.

\section{Laparoscopic sterilisation occlusion procedures}

Sterilisation using mechanical devices represents the preferred and recommended method of sterilisation. ${ }^{3}$ Of the mechanical devices available, the Falope ring, Hulka clip and Filshie clip are the most commonly used. The Falope ring (also called the Yoon ring after its developer) is a silicone rubber band that is fitted around a loop in the Fallopian tube, thereby making it a more technically challenging procedure compared with the application of clips. Reports have suggested that there is more postoperative pain with this method than with clips. ${ }^{15}$ In addition, although the ring destroys about $3 \mathrm{~cm}$ of tube, reversal results appear to be satisfactory.

The surgical procedure for the Hulka and Filshie clips entails placement of the clip on the mid-isthmic portion of the Fallopian tube (Figure 1). The Hulka clip is a hinged clip made of two toothed jaws of Lexan ${ }^{\circledR}$ plastic joined by a stainless steel hinge pin. A gold-plated stainless steel spring is pushed from behind onto the jaws to maintain pressure and keep the jaws closed.

The Filshie clip represents the most recent mechanical device and received Food and Drug Administration (FDA) approval in 1996. The Filshie clip is made of titanium and lined with silicon rubber. Following placement on the Fallopian tube, the upper curved jaw is compressed with an applicator so that the upper jaw is locked onto the lower jaw (Figure 1). Flattening the upper jaw compresses the rubber, thereby occupying any space made by the compressed tube. ${ }^{16}$ As tubal necrosis occurs, the silicone rubber expands to maintain blockage of the lumen. Because the silicone rubber of the Filshie clip is able to expand and provide continuous pressure, any residual tubal patency, such as may occur with the spring clip, is prevented. Furthermore, since only approximately $4 \mathrm{~mm}$ of Fallopian tube is destroyed with the clips, the chances of successful reversal are enhanced with these techniques. ${ }^{17}$

\section{Hysteroscopic sterilisation occulsion procedures}

Hysteroscopic sterilisation using the Essure and Adiana devices can usually be performed on a day care basis, and since there is no abdominal incision little or no anaesthetic is required. In October 2002, Essure received FDA approval, representing the first transcervical hysteroscopically placed sterilisation method. The Essure device is a rod that is covered with an expandable spring of nitinol. The device is inserted into the Fallopian tube and induces scar tissue to form over and into the implant, blocking the Fallopian tube and preventing fertilisation of the egg by the sperm. The method is irreversible as the device becomes intimately involved with tissues of the interstitial and early isthmic portion of the Fallopian tube.

The Adiana technique involves two steps: first, radiofrequency energy is applied to the Fallopian tubes to denude the tubal mucosal lining. This acts to destroy the epithelial cells, preventing re-canalisation of the tube and facilitating wound healing and motility of the underlying tubal tissue. In the second step a porous, non-biodegradable matrix plug is inserted into the cauterised lumen, which acts as a scaffold for infiltrating interstitial healing tissue and thereby irreversibly occludes the tube. Both the Essure and Adiana devices require specifically trained and experienced hysteroscopists. Women must also undergo a hysterosalpingogram approximately 3 months later in order to confirm proper device placement and occlusion of the Fallopian tubes.

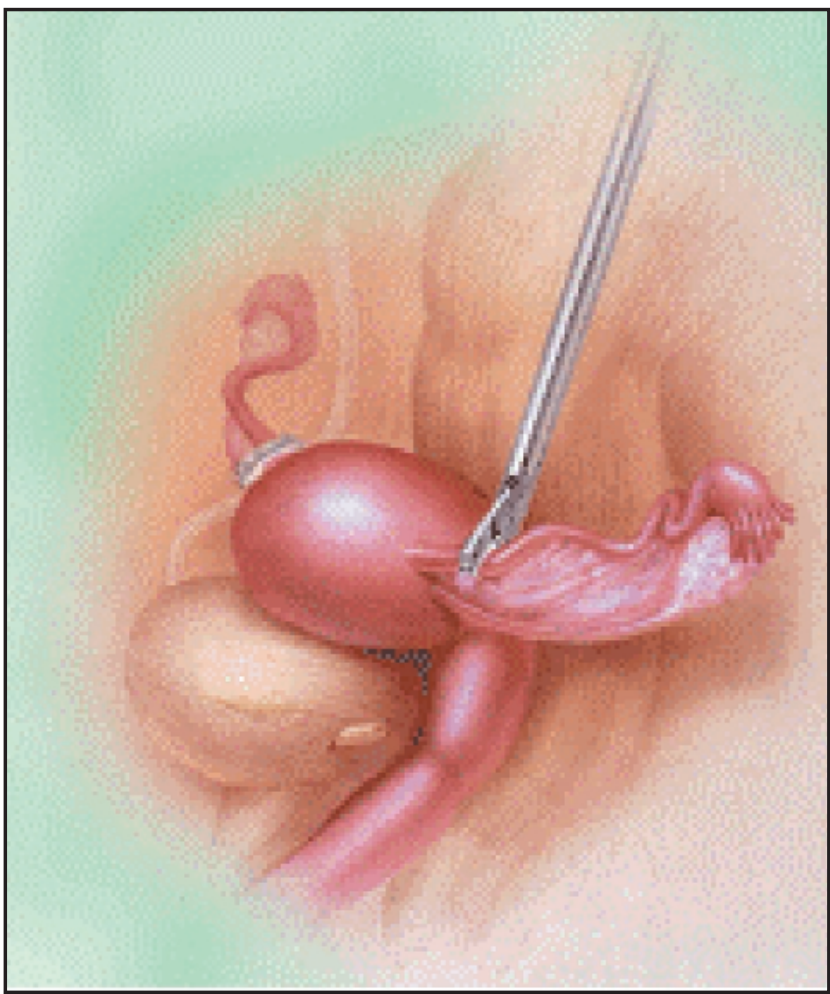

Figure 1 Diagrammatic representation of laparoscopic sterilisation techniques with the Filshie clip. Figure (C) Femcare-Nikomed Ltd, and reproduced with the kind permission of the copyright owner 


\section{Guidelines, counselling and failure risk}

As well as the usual history and examination, counselling is a crucial element in the decision to undergo female sterilisation. In order that women can make informed decisions about their choice of long-term contraception, unbiased accurate information should be provided, including information on the range of other methods available, the procedures involved and their benefits, risks and possible complications. Male sterilisation must also be offered as an alternative. For women wishing to undergo sterilisation, it is essential to provide sufficient information in order to reduce regret at a later stage. The two most common factors associated with regret are young age and unpredictable life events, such as change in marital status or death of a child.7,18

In the UK, the RCOG recently updated its evidencebased guidelines for male and female sterilisation. ${ }^{3}$ The aim of these guidelines is to ensure that patients receive a high-quality service based on available evidence and expert opinion. Accordingly, the guidelines state that women should be informed about the lifetime risk of failure in general for tubal occlusion, which is estimated at 1 in 200 . The longest period of follow-up data available for the most common method used in the UK, the Filshie clip, suggests a failure rate after 10 years of $2-3$ per 1000 procedures. In addition, women should be counselled on the potential irreversibility of the procedure, the small risk of ectopic pregnancy if the procedure fails, and the risk of laparotomy as a result of severe complications, which has been reported as 1.9/1000 in a large prospective study and as $1.4-3.1 / 1000$ cases in two other practice surveys. ${ }^{3}$ In terms of methods, the RCOG guideline recommends mechanical occlusion of the tubes by either Filshie clips or rings as the method of choice for laparoscopic tubal occlusion.

\section{Clinical data assessment}

When assessing the efficacy of sterilisation techniques, a minimum 2-year follow-up is recommended, although longer follow-up is preferable. Sterilisation failure is generally expressed as a lifetime risk, whereas reversible method failures are expressed as a Pearl index, since they can occur at any subsequent year of use. To date, there are few data relating to long-term failure rates following female sterilisation.

When assessing long-term data a number of key considerations should be taken into account as follows:

- Studies should be interpreted in the context of all available data.

- Failure rates should be considered in conjunction with the safety and morbidity as well as acceptability of the procedures evaluated.

- Where large statistically powered studies are unavailable, all data need to be carefully assessed in order that informed decisions can be made on appropriate treatments.

- Differences in the definition of intention-to-treat populations need to be considered when evaluating data.

- Finally, as well as being simple, effective and safe, assessment of the cost-effectiveness of procedures needs to be taken into account.

\section{Long-term efficacy data for female sterilisation \\ Comparative data for different methods of female sterilisation}

The US Collaborative Review of Sterlization (CREST) study was a prospective, multicentre, observation trial conducted by the Centers for Disease Control and

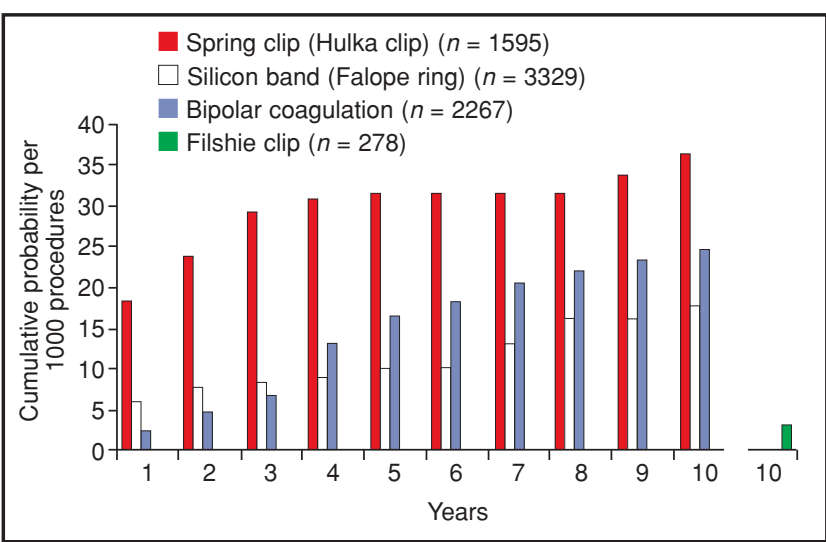

Figure 2 Cumulative probability of pregnancy among women for the CREST study ${ }^{19}$ using the spring clip $(n=1595)$, the silicon band $(n=3329)$ and bipolar coagulation $(n=2267)$ and the Filshie clip study based on prospective data for the 278 patients included in the 10-year follow up $(n=278)^{32}$

Prevention, which assessed the long-term risks of various sterilisation methods in a large cohort of 10685 women. ${ }^{19}$ The methods included laparoscopic unipolar cautery, bipolar cautery, Hulka clip and Falope ring application and postpartum partial salpingectomy (mainly Pomeroy technique). Ten-year follow-up data from this study showed that the failure rate was $2.48 \%$ for bipolar cautery, $3.65 \%$ for Hulka clip sterilisation and $1.77 \%$ for the Falope ring (Figure 2). The lowest rates were observed in patients after unipolar coagulation or postpartum partial salpingectomy $(0.75 \%)$. For those patients under the age of 28 years, the failure rate was even higher, with rates as high as $5.2-5.4 \%$ for bipolar cautery and Hulka clips. As the Filshie clip had not been introduced into clinical practice when the study was conducted, data on its efficacy were not included as part of the CREST study.

The observed failure rates in the CREST study were surprisingly high and have influenced the overall perception of the efficacy of female sterilisation. Moreover, the CREST data are usually used as part of the counselling for comparability of the efficacy of female sterilisation (including that of the Filshie clip) with the IUS, even though the CREST study did not include data on the Filshie clip. 20,21 Indeed, at the RCOG Meeting in Cairo it was acknowledged (by Schering and Professor Luukkainen in the Schering-sponsored symposium) that when stating that the levonorgestrel (LNG) IUS is equivalent to female sterilisation, they are basing this on the CREST data and not Filshie clip data. Therefore, it is important that efficacy data are placed in context with all other available data, including those of the Filshie clip.

Other studies assessing the failure rate of the LNG IUS have reported a Pearl index of 0.18 based on a 7-year randomised study. ${ }^{22}$ However, it is essential to take into account the failure rate of the entire intention-to-treat population. Accordingly, for the IUS, the long-term pregnancy rates should take into consideration those patients in whom the IUS was expelled and those patients in whom the IUS was removed due to adverse effects. Over a 5-year period, approximately $5.9 \%$ of LNG IUS were spontaneously expelled from the body, representing a premature failure of the method. ${ }^{23}$ In addition, a large number, varying from $30 \% 24$ to $45 \% 25$ of LNG IUS needed to be removed as a result of adverse side effects. In particular, abnormal bleeding represents a significant problem with the IUS; ${ }^{26}$ however, following the first initial troublesome months, the reduction in the amount of menstrual bleeding and in the number of days of menstrual bleeding makes the IUS suitable for the treatment of menorrhagia. 


\section{Filshie clip}

The Filshie clip system represents one of the most popular and preferred methods of female surgical contraception used by surgeons. ${ }^{27,28}$ Studies with the Filshie clip have demonstrated a failure rate of $0.27 \%$ (at 2-year followup). ${ }^{29}$ Long-term studies with the Filshie clip have confirmed these low failure rates. ${ }^{30-32}$ In particular, a recent retrospective questionnaire-based study conducted in Australia examining 30000 applications of the Filshie clip showed a $99.6 \%$ response rate and an overall failure rate of $2-3$ per 1000.30

Currently, there are few data relating to laparoscopic sterilisation during the postpartum period. ${ }^{33,34}$ In 1990, a study by Yan et al. of 200 women compared the Filshie clip and Pomeroy technique in the postpartum state. After a 2-year follow-up, only one pregnancy occurred, which occurred 6 months after surgery in a patient in the Pomeroy group. ${ }^{34}$ More recently, Najia et al. reported on a retrospective evaluation of the Filshie clip technique to determine whether the laparoscopic procedure is a safe and reliable postpartum technique. ${ }^{35}$ In all 84 cases examined, the procedure was completed successfully, with no injuries to any internal organs and no known failures reported to date, demonstrating the safety and reliability of this technique in experienced hands.

Because of the low failure rates with sterilisation, a large number of patients are required to allow appropriate statistical comparisons between the Filshie clip and other methods of sterilisation. However, Filshie clip data support the use of this device as a preferred method of female sterilisation (Figure 2).32 Importantly, these patient populations include failures that occurred as a result of operator failure, including tubal non-occlusion or wrong structure application. ${ }^{4}$ If the criteria reported for the LNG IUS were applied to these patients and those with operator failure were excluded, a marked reduction in overall 10-year failure rate from $0.56 \%$ to $0.2 \%$ would be observed. ${ }^{36}$ These data highlight the importance of considering all patients in the intention-to-treat group and the bias in results that might be generated if the population criteria are not apparent. These findings also stress the importance of appropriate training since, when performed using the correct technique, failure rates with Filshie clips are extremely low.

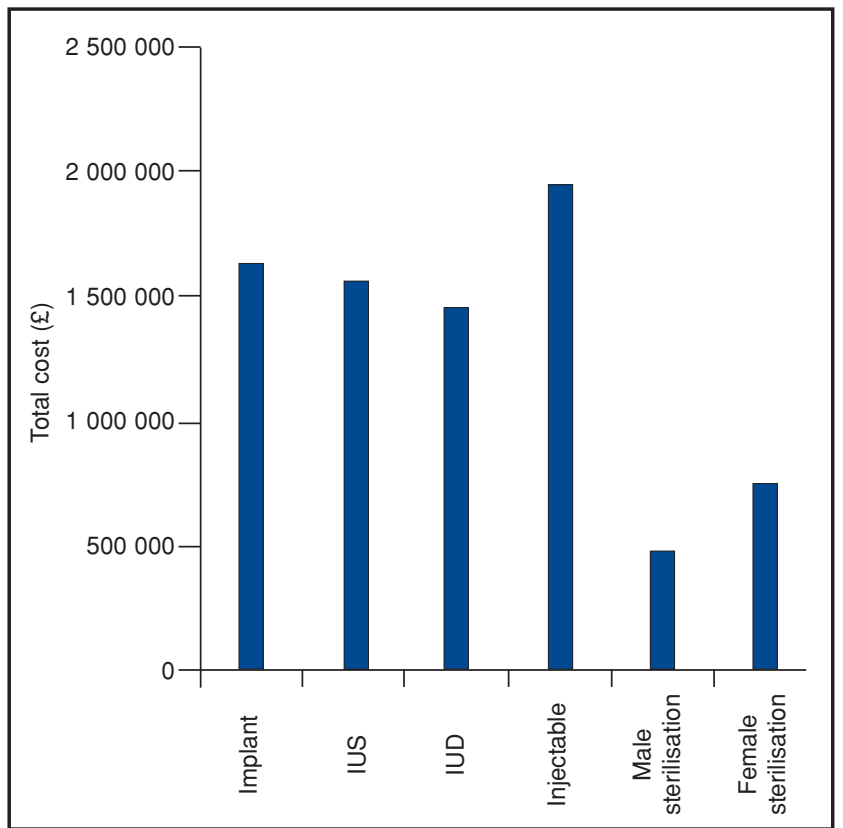

Figure 4 Cost-effectiveness of sterilisation versus long-term contraception following 15 years of contraceptive protection. ${ }^{1}$ IUD, intrauterine device; IUS intrauterine system

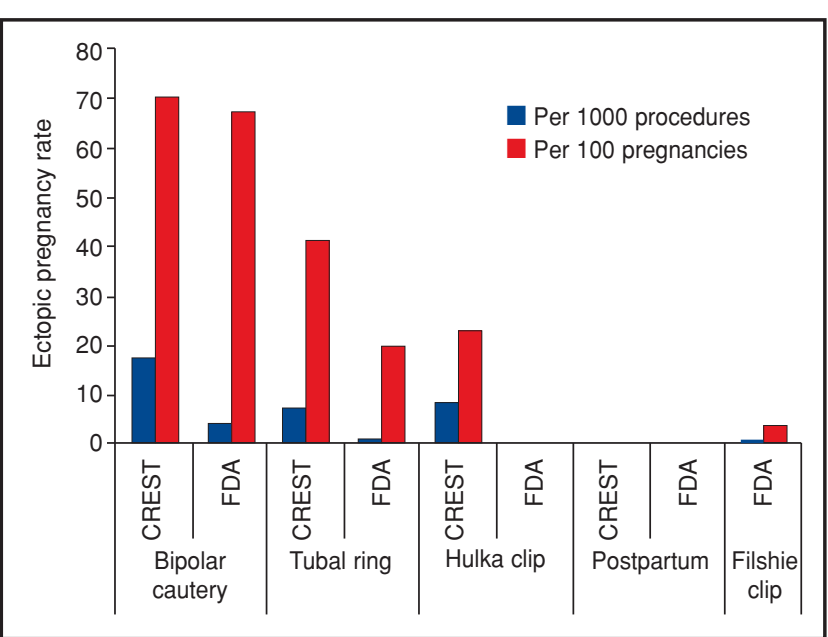

Figure 3 Ectopic pregnancy rates versus sterilisation method used $^{29,38}$

\section{Hysteroscopic techniques}

Essure and Adiana present promising techniques based on clinical data. ${ }^{37}$ Importantly, however, efficacy data focus only on the patient population in which correct placement has occurred. Bilateral placement rates for Essure have been reported as being between $81 \%$ and $85 \%$, with patient satisfaction of approximately $94 \%$ (with responses as 'very' or 'somewhat' satisfied). ${ }^{11,37}$ Research around Adiana is ongoing, but interim clinical trial results have indicated a bilateral first attempt access rate of $94.5 \% .^{12}$

\section{Safety data}

Due to their more favourable safety profile, mechanical devices are preferred methods to electrocautery for female sterilisation. In addition to having a lower failure rate compared with bipolar cautery, rings and clips are also associated with fewer ectopic pregnancies (Figure 3). Data from the CREST study showed an ectopic pregnancy rate of $67 \%$ in those patients in whom the method failed, giving an overall ectopic pregnancy rate of $1-2 \%$ of all sterilisations with bipolar cautery. ${ }^{38}$ In contrast, mechanical methods have a much lower incidence of ectopic pregnancy of approximately $4 \% .38$ In particular, ectopic pregnancy with the Filshie clip only occurs in $4 \%$ of failures. ${ }^{29,38}$ In the absence of long-term data with the Filshie clip, a theoretical value for the ectopic pregnancy rate with the Filshie clip can be determined by applying the $4 \%$ incidence of ectopic pregnancy to the failure rate observed with the Filshie clip (i.e. 2-3/1000 patients), giving a rate of less than 1 in 6000 . Although this value is only an estimate, the Filshie clip may protect against ectopic pregnancy. Reversal of clip sterilisation is generally accepted as having a high success rate $(80-100 \%)$ compared with other methods; however, it should be made clear to patients that reversal involves minilaparotomy, does not always succeed, and carries a risk of ectopic pregnancy (up to 5\%).17,39,40

Because of the high failure rate of diathermy and the ectopic failure rate, we believe that this procedure is extremely dangerous and should never replace mechanical devices for financial reasons. Rather we would recommend that for countries with financial limitations, physicians should try to obtain donor clips or donor rings whenever possible.

It should be noted that patients who are overweight and/or with previous abdominal surgery represent a high-risk population for female sterilisation. If laparoscopy is performed on this high-risk population, it should always be by an experienced surgeon, and in most cases patients should be recommended alternative contraceptive procedures. 


\section{Cost-effectiveness}

In addition to efficacy and safety, recent data have examined the cost-effectiveness of different contraceptive methods. In particular, NICE has reported on the costeffectiveness of LARC methods (i.e. implant, IUS, IUDs and injectables) when compared with the combined oral contraceptive pill, the male condom, and female and male sterilisation. ${ }^{1}$ Obvious differences in their use will impact on cost-effectiveness, including daily administration of the oral contraceptive pill, use of condoms at every intercourse and IUDs lasting a minimum of 5 years. The findings of this analysis showed that all LARC methods are more costeffective than the combined oral contraceptive pill because accidental pregnancy is less likely. In addition, this analysis showed that at 15 years of contraceptive use, female and male sterilisation are more cost effective than all the LARC methods (Figure 4).

\section{Conclusions}

Over the last few decades a number of mechanical devices have been introduced for use in female sterilisation, including the Falope ring, Hulka clip and Filshie clip. These devices have revolutionised sterilisation with the result that serious complications using this method are rare.

To date, few comparative long-term studies for female sterilisation exist, and it is important that all data are evaluated so that women can make informed decisions about long-term contraception. To this end, it is important that patients receive valid counselling information related to the specific sterilisation method to be used. In particular, failure rates should be considered in the context of all available data and in conjunction with the safety, acceptability and cost-effectiveness of the procedures evaluated.

The CREST study revealed cumulative 10-year failure rates higher than previously thought; nevertheless the findings of the CREST study confirm that sterilisation, when performed using the appropriate technique by an experienced clinician, is an extremely effective long-term contraceptive method. The CREST study did not include the Filshie clip, which has been shown to have extremely low failure rates (i.e. 2-3 per 1000) and accordingly this device is now recognised to be a method of choice for female sterilisation. ${ }^{3}$ In addition to its use in female sterilisation, the Filshie clip has also been used as a surgical occlusive device in general surgery and in gynaecological surgery. Such procedures include laparoscopic cholecystectomy, appendectomy, ectopic pregnancy, oophorectomy following hysterectomy and bladder neck colposuspension. ${ }^{41}$

In conclusion, with the increasing demand for effective long-term contraception, it is important that safer, easier and more cost-effective techniques continue to be developed. Improvements in surgical techniques continue to evolve, with hysteroscopic methods now available and future research investigating the use of microlaparoscopy and disposable applicators.

\section{Statements on funding and competing interests}

\section{Funding None identified.}

Competing interests All the authors received funding from Femcare-Nikomed Ltd to attend and present at the 6th International Scientific Meeting of the Royal College of Obstetricians and Gynaecologists in Cairo, Egypt in September 2005.

\section{References}

1 National Institute for Health and Clinical Excellence (NICE). Long-acting Reversible Contraception (CG30). http://www.nice. org.uk/CG030NICEguideline [Accessed 4 March 2007].

2 United Nations. Levels and Trends of Contraceptive Use as Assessed in 1998. New York, NY: Population Division, Department of Economic and Social Affairs, United Nations, 1998.
3 Royal College of Obstetrician and Gynaecologists. Male and Female Sterilisation (Evidence based Clinical Guideline No. 4). 2004. http://www.rcog.org.uk/resources/Public/pdf/sterilisation full060607.pdf [Accessed 4 March 2007].

4 Varma R, Gupta JK. Failed sterilisation: evidence-based review and medico-legal ramifications. Br J Obstet Gynaecol 2004; 111: 1322-1332.

5 General Housekeeping Survey (Series FMI No. 24). London, UK: Office of National Statistics, 1997.

6 Bishop EN, Nelms WF. A simple method of tubal sterilisation. $N$ Y State J Med 1930; 39: 214-216.

7 Pati S, Cullins V. Female sterilization. Evidence. Obstet Gynecol Clin North Am 2000; 27: 859-899.

8 Jansen FW, Kapiteyn K, Trimbos-Kemper T, Hermans J, Trimbos JB. Complications of laparoscopy: a prospective multicentre observational study. Br J Obstet Gynaecol 1997; 104: 595-600.

9 Chapron C, Querleu D, Bruhat MA, Madelenat P, Fernandez H, Pierre $F$, et al. Surgical complications of diagnostic and operative gynaecological laparoscopy: a series of 29,966 cases. Hum Reprod 1998; 13: 867-872.

10 Sciarra J, Butler J, Speidel J (eds). Hysteroscopic Sterilisation. New York, NY: Intercontinental Medical Books, 1974.

11 Kerin JF, Carignan CS, Cher D. The safety and effectiveness of a new hysteroscopic method for permanent birth control: results of the first Essure pbc clinical study. Aust N Z J Obstet Gynaecol 2001; 41: 364-370.

12 Vancaille TG, Herbst S, Harris M, Cooper JM, Galen DI, Zimmerman J. The Adiana procedure: early results of the EASE clinical trial. J Am Assoc Gynecol Laparosc 2003; 10: 56.

13 de Villiers VP. Postpartum sterilisation with the Filshie titanium silicone-rubber clip and subsequent pregnancy. S Afr Med $J$ 1987; 71: 498-499.

14 Chi IC, Mumford SD, Gardner SD. Pregnancy risk following laparoscopic sterilization in nongravid and gravid women. $J$ Reprod Med 1981; 26: 289-294.

15 Dobbs FF, Kumar V, Alexander JI, Hull MG. Pain after laparoscopy related to posture and ring versus clip sterilization. Br J Obstet Gynaecol 1987; 94: 262-266.

16 Filshie M. Laparoscopic sterilization. Semin Laparosc Surg 1999; 6: 112-117.

17 Grimes D. Update on female sterilisation. Contracept Rep 1997; 7: 13.

18 Hillis SD, Marchbanks PA, Tylor LR, Peterson HB. Poststerilization regret: findings from the United States Collaborative Review of Sterilization. Obstet Gynecol 1999; 93: 889-895.

19 Peterson HB, Xia Z, Hughes JM, Wilcox LS, Tylor LR, Trussell $J$. The risk of pregnancy after tubal sterilization: findings from the U.S. Collaborative Review of Sterilization. Am J Obstet Gynecol 1996; 174: 1161-1168, discussion 1168-1170.

20 Faculty of Family Planning and Reproductive Health Care (FFPRHC) Clinical Effectiveness Unit. FFPRHC Guidance (April 2004). The levonorgestrel-releasing intrauterine system (LNG-IUS) in contraception and reproductive health. J Fam Plann Reprod Health Care, 2004; 30: 99-109.

21 Mattinson A, Mansour D. Female sterilisation: is it what women really want? J Fam Plann Reprod Health Care 2003; 29: 136-139.

22 Sivin I, Stern J, Coutinho E, Mattos CE, el Mahgoub S, Diaz S, et al. Prolonged intrauterine contraception: a seven-year randomized study of the levonorgestrel $20 \mathrm{mcg} /$ day $(\mathrm{LNg} 20)$ and the Copper T380 Ag IUDS. Contraception 1991; 44: 473-480.

23 Cox M, Tripp J, Blacksell S. Clinical performance of the levonorgestrel intrauterine system in routine use by the UK Family Planning and Reproductive Health Research Network: 5-year report. J Fam Plann Reprod Health Care 2002; 28: 73-77.

24 Andersson K, Odlind V, Rybo G. Levonorgestrel-releasing and copper-releasing (Nova T) IUDs during five years of use: a randomized comparative trial. Contraception 1994; 49: 56-72.

25 Backman T, Huhtala S, Blom T, Luoto R, Rauramo I, Koskenvuo M. Length of use and symptoms associated with premature removal of the levonorgestrel intrauterine system: a nation-wide study of 17,360 users. Br J Obstet Gynaecol 2000; 107: 335-339.

26 Backman T. Benefit-risk assessment of the levonorgestrel intrauterine system in contraception. Drug Saf 2004; 27 1185-1204.

27 Garrud P, Sheard CE, Filshie G, Beattie A. Effective female sterilisation: a survey of UK gynaecologists' practices. CME Bulletin Gynaecology 2000; 12: 13-17.

28 Penney GC, Souter V, Glasier A, Templeton AA. Laparoscopic sterilisation: opinion and practice among gynaecologists in Scotland. Br J Obstet Gynaecol 1997; 104: 71-77. 
29 FDA Advisory Board Meeting. Presentation made by Professor Theodore King, 26 February. 1996.

30 Kovacs GT, Krins AJ. Female sterilisations with Filshie clips: what is the risk failure? A retrospective survey of 30,000 applications. J Fam Plann Reprod Health Care 2002; 28: 34-35.

31 Puraviappen AP, Hamid Arshat A. Experiences with the Filshie clip sterilisation. Adv Contracept 1987; 3: 13-17.

32 Filshie G. Long-term experience with the Filshie Clip. Gynaecology Forum 2002; 7: 7-10.

33 Filshie G. Postpartum use of the Filshie clip for female sterilisation. Adv Contracept 1987; 3: 175-5.

34 Yan JS, Hsu J, Yin CS. Comparative study of Filshie clip and Pomeroy method for postpartum sterilization. Int J Gynaecol Obstet 1990; 33: 263-267.

35 Najia SK. Post-partum female sterilisation. Poster presentation at the XII Annual Meeting of the International Society for Gynecologic Endoscopy, Cancun, Mexico, 31 March-7 April 2003.

36 Penfield AJ. The Filshie clip for female sterilization: a review of world experience. Am J Obstet Gynecol 2000; 182: 485-489.
37 Duffy S, Marsh F, Rogerson L, Hudson H, Cooper K, Jack S, et al. Female sterilisation: a cohort controlled comparative study of ESSURE versus laparoscopic sterilisation. $\mathrm{Br} J$ Obstet Gynaecol 2005; 112: 1522-1528.

38 Peterson HB, Xia Z, Hughes JM, Wilcox LS, Tylor LR, Trussell $J$. The risk of ectopic pregnancy after tubal sterilization. U.S. Collaborative Review of Sterilization Working Group. N Engl J Med 1997; 336: 762-677.

39 Hulka JF, Noble AD, Letchworth AT, Lieberman B, Owen E, Gomel V, et al. Reversibility of clip sterilizations. Lancet 1982; 2: 927.

40 Nwagbara PN, Stibbe HM, Browning AJ, Tonks AM. Reversal of female sterilisation experience in a district general hospital. $J$ Obstet Gynaecol 1997; 17: 293-297.

41 Yehia M. A fresh perspective on the use of the Filshie clip. Keynote speech made at the 'The changing face of female sterilization: meeting the needs of the 21st century woman symposium at the 6th International Scientific Meeting of the Royal College of Obstetricians and Gynaecologists, Cairo, Egypt, 2005.

\section{JOURNAL REVIEWS}

Cancer risk among users of oral contraceptives: cohort data from the Royal College of General Practitioners' oral contraception study. Hannaford PC, Selvaraj S, Elliott AM, Angus V, Iversen L, Lee AJ. BMJ 2007; 335: 651-654

The latest report from this large cohort study includes over a million years of observation, accumulated over 36 years. The advantage of reporting at this stage is that many women in the cohort study are now postmenopausal and at an age when cancers are more common.

When compared with the 339000 neverusers of oral contraception, the incidence of cancers among 744000 ever-users was significantly lower for colorectal, uterine body and ovarian cancers. There was a non-significant increased risk of cervical cancer, which was unaffected by adjusting for smoking and other potential confounders. The risk of breast cancer was not increased [relative risk (RR) 0.98, CI $0.87-1.10]$ and the risk of any cancer was significantly reduced (RR 0.88 , CI $0.83-0.94$ ).

Information on type and duration of oral contraceptives used was obtained from a smaller subset of women. Long-term use (i.e. $>8$ years) of oral contraception was associated with a significantly reduced risk of ovarian and uterine body cancer and a significantly increased risk of cervical cancer. Both the protective effect on ovarian cancer and the excess risk of cervical cancer persisted $10-15$ years after stopping.

One unexpected finding was an increased incidence of brain or pituitary cancers (RR 5.51, CI 1.38-22.05). The number of tumours was small and the confidence interval is wide so the risk is likely to be of low clinical significance if it exists at all

The findings of this study are largely reassuring and they are remarkably consistent with those of the Oxford Family Planning Association ${ }^{1}$ and Luie et al. ${ }^{2}$ previously reviewed in this journal. Thus the conclusions are likely to be valid despite potential bias from the large losses to follow-up and changes in estrogen dose with time. Patients alarmed by the CNS tumour data should be reminded that suggestions of a link between mobile phones and brain tumours have not caused them to throw away their phones, so they should think twice about throwing away their pills.

Reviewed by Louise Melvin, MRCOG, MFFP Subspecialty Specialist Registrar, Family Planning \& Well Woman Clinic, Edinburgh, UK

\section{References}

Vessey M, Painter R. Oral contraceptive use and cancer. Findings in a large cohort study, 1968-2004. $J$ Cancer 2006; 95: 385-389.

2 Luie G, Thompson P, McDuffie KE, Carney ME, Terada KY, Goodman MT. Association of estrogen and progestin potency of oral contraceptives with ovarian carcinoma risk. Obstet Gynecol 2007; 109: 597-607.

Contraceptives and the risk of death from breast cancer. Wingo PA, Austin $\mathrm{H}$, Marchbanks P, Whiteman MK, Hsia J, Mandel MG, et al. Obstet Gynecol 2007; 110: 793-800

The oral contraceptive (OC) pill remains one of the most popular means of contraception worldwide. Previously, recent OC use had been shown to be associated with a slightly highe breast cancer incidence amongst younge women. ${ }^{1}$ However, the Royal College of Genera Practitioners' oral contraception study contradicts these findings. It showed no difference in the incidence of breast cancer between never-user and ever-users of OCs. ${ }^{2}$ The impact of OC use on survival after diagnosis of breast cancer is not known.

The aim of the study by Wingo et al. was to examine the relationship between OC use and death from breast cancer over a 15-year followup period. It linked data from the CASH (Cancer and Steroid Hormone) study with mortality data from the Surveillance, Epidemiology and End Results Program. The CASH study was a large, American, population-based, case-control study designed to examine the risks of OCs and breast ovarian and endometrial cancers. ${ }^{3}$ Women aged 20-54 years with histologically confirmed primary breast cancer between 1980 and 1982 were interviewed 1-31 (mean, 12) weeks after diagnosis. OC use in this study was shown not to be associated with a higher incidence of breast cancer development. Over $95 \%$ of interviews were successfully linked to the cancer registry data from the Surveillance, Epidemiology and End Results Program.

A total of 4292 women were included in this study; 1473 died of breast cancer during the follow-up period. Survival rates were $80 \%$ at 5 years and $64 \%$ at 15 years. This correlates with current UK breast cancer mortality statistics. ${ }^{4}$ There was no association between mortality and duration of OC use, pill potency, age at first use or time since first use. The risk of death decreased significantly with increasing time since last use but there was no consistent gradient effect. The overall conclusion was that there was no evidence of either benefit or harm of prior OC use on longterm survival after diagnosis of breast cancer. The main limitation of the study is that the findings are based only on risk factors reported during the initial interview after diagnosis. The study was unable to provide information on hormone receptor status or genetic factors such as BRCA1 or BRCA2 status, or indeed on new or continued OC use after diagnosis. However, there was a long follow-up period with a very low loss to follow-up (less than 3\%), which makes the key findings particularly reassuring.

Reviewed by Shazia Bhatti, MRCOG Specialist Registrar in Obstetrics and Gynaecology, Luton and Dunstable Hospital NHS Foundation Trust, Luton, UK

\section{References}

Breast cancer and hormonal contraceptives: collaborative reanalysis of individual data on 53297 women with breast cancer and 100239 women with Collaborative Group on Hormonal Factors in Breast Collabor

t AM, Angus V, Iversen $L$, Lee AJ Cancer risk among users of oral , Lee AJ. Cancer risk among users of oral contraceptives: cohort data from the Royal College of 2007; 335: 651-654.

Wingo PA, Webster LA, Ory HW. Oral contraceptive use and the risk of breast cancer. N Engl J Med 1986; 315: $405-411$.

Coleman MP, Rachet B, Woods LM, Mitry E, Riga M, Cooper N, et al. Trends and socioeconomic inequalities in cancer survival in England and Wales up to 2001. Br J Cancer 2004; 90: 1367-1373.

\section{PEER REVIEWERS}

If you have a special interest in one or more of the topics covered by the Journal and have some time available to peer review occasional papers in your own area(s) of expertise then perhaps you might be interested in joining the Journal's team of peer reviewers? In common with the majority of other academic journals, peer reviewers offer their services on a voluntary basis; however, if you are a member of the Faculty of Sexual and Reproductive Healthcare then each completed review counts for two (2) CME points. For further information please contact the Journal Editorial Office at journal@fsrh.org. 